\title{
Erratum to: Liver stiffness measurement by acoustic radiation force impulse is useful in predicting the presence of esophageal varices or high-risk esophageal varices among patients with HCV-related cirrhosis
}

\author{
Naoki Morishita $\cdot$ Naoki Hiramatsu $\cdot$ Tsugiko Oze $\cdot$ Naoki Harada \\ Ryoko Yamada - Masanori Miyazaki - Takayuki Yakushijin - Takuya Miyagi • \\ Yuichi Yoshida · Tomohide Tatsumi · Tatsuya Kanto · Tetsuo Takehara
}

Published online: 4 April 2015

(C) Springer Japan 2015

Erratum to: J Gastroenterol (2014) 49:1175-1182

DOI 10.1007/s00535-013-0877-z

The original version of this article unfortunately contained errors in Table 1. Table 1 should be shown as follows.
The online version of the original article can be found under doi:10.1007/s00535-013-0877-z.

N. Morishita $(\bowtie) \cdot$ N. Hiramatsu $\cdot$ T. Oze $\cdot$ N. Harada R. Yamada $\cdot$ M. Miyazaki $\cdot$ T. Yakushijin · T. Miyagi · Y. Yoshida $\cdot$ T. Tatsumi - T. Takehara

Department of Gastroenterology and Hepatology, Osaka

University Graduate School of Medicine, 2-2 Yamadaoka, Suita,

Osaka 565-0871, Japan

e-mail: n.morishita@gh.med.osaka-u.ac.jp

N. Hiramatsu

e-mail: hiramatsu@gh.med.osaka-u.ac.jp

T. Kanto

Department of Gastroenterology and Hepatology, National

Center for Global Health and Medicine, Ichikawa, Japan
Table 1 Baseline characteristics of patients in training and validation sets

\begin{tabular}{lll}
\hline Factor & Training set & Validation set \\
\hline Number & 92 & 43 \\
Age (year) & $68.7 \pm 9.3$ & $72.6 \pm 6.9$ \\
Sex (male/female) & $47 / 45$ & $26 / 17$ \\
BMI $\left(\mathrm{kg} / \mathrm{m}^{2}\right)$ & $22.3 \pm 3.2$ & $22.3 \pm 3.2$ \\
Child-Pugh class & & \\
A & $60(65.2 \%)$ & $31(72.1 \%)$ \\
B & $30(30.3 \%)$ & $11(25.6 \%)$ \\
C & $2(2.0 \%)$ & $1(2.3 \%)$ \\
HCC & & \\
No & $46(50.0 \%)$ & $12(27.9 \%)$ \\
Yes & $46(49.0 \%)$ & $31(72.1 \%)$ \\
Ascites & & \\
No & $81(88.0 \%)$ & $35(81.4 \%)$ \\
Yes & $11(12.0 \%)$ & $8(18.6 \%)$ \\
Platelet $\left(\times 10^{4} / \mathrm{mm}^{3}\right)$ & $9.64 \pm 4.96$ & $9.85 \pm 4.30$ \\
AST $(\mathrm{IU} / \mathrm{L})$ & $54.3 \pm 30.0$ & $38.6 \pm 22.6$ \\
ALT $(\mathrm{IU} / \mathrm{L})$ & $49.2 \pm 33.0$ & $53.4 \pm 38.6$ \\
Albumin $(\mathrm{g} / \mathrm{dl})$ & $3.59 \pm 0.59$ & $3.67 \pm 0.56$ \\
Total bilirubin $(\mathrm{mg} / \mathrm{dl})$ & $1.07 \pm 0.86$ & $1.02 \pm 0.61$ \\
\hline Res & &
\end{tabular}

Results are given as mean \pm standard deviation or $n(\%)$

$B M I$ body mass index, $H C C$ hepatocellular carcinoma, $A S T$ aspartate aminotransferase, $A L T$ alanine aminotransferase 